1265). (Reprints: Prof Graham Harding, Neurosciences Research Institute, Aston University, Aston Triangle, Birmingham B4, UK).

COMMENT. The specific vigabatrin-induced visual field loss is a bilateral concentric constriction with binasal annular defect and relative temporal sparing. Central vision is usually unaffected by vigabatrin. Vigabatrin is sometimes advocated in the treatment of West syndrome, a condition with onset in infancy. Since the test described for detecting visual field defects was applied to children between 3 and 15 years, presumably it would not be appropriate for the majority of infants treated. The $25 \%$ risk of visual field loss is of serious concern in the continued use of vigabatrin for infantile spasms, despite the ability to identify the defect in younger children.

\title{
SEIZURES AND MUNCHAUSEN SYNDROME BY PROXY
}

The prevalence, morbidity and mortality, diagnosis and management of cases of fabricated seizures and child abuse (Munchausen syndrome by proxy (MShP)) are assessed by pediatricians at the University of Wales College of Medicine, Cardiff, UK. A survey of pediatric neurologists in the USA found that 107 of 190 respondees (21.8\% return rate) reported contact with at least one case of Munchausen by proxy (Schreier HA, Libow JA. 1993), and seizures are a presenting feature in $42 \%$ of MSbP cases (Rosenberg DA. 1987). Meadow R (1984) cites seizures as the most common example of MSbP in children. Death rates are generally quoted at $10 \%$ (Rosenberg, 1987). Surviving children adopt a self image as disabled persons. The most vulnerable are children under 5 years of age, some deaths being ascribed to sudden unexpected death in epilepsy (SUDEP). Perpetrators, usually mothers, are frequently hostile and aggressive toward the physician and nurses, a reaction that may be a deterrent to making the diagnosis of MSbP. The pediatrician needs to communicate with and recruit the family doctor for opinions regarding the prior history of the child and family. Practical guidelines to avoid a false diagnosis of epilepsy include prolonged EEG or videoEEG. An early diagnosis of MSbP is supported by a temporal relation between symptoms and mother's presence, a father's opposing opinion regarding symptoms, covert video surveillance of hospitalized patient, and meticulous records for possible court involvement. Avoid unnecessary escalation of medical treatment by maintaining a healthy suspicion of MSbP in cases inat are unexpectedly unresponsive to adequate therapy, frequent unexplained low antiepileptic drug levels and obvious non-compliance, lack of seizure recurrence during carefully monitored drug withdrawal, and mother's insistence on further tests and consultations. Appropriate management may include a psychiatric opinion regarding the parent-child dynamic, a shift of emphasis in management from child to mother or carer, involvement of social service professionals, a period of observation in foster care, and commitment to follow-up by expert in child protection. Fabricated seizures require particular attention from epileptologists, pediatric neurologists, and pediatricians. (Barber MA, Davis PM. Fits, faints, or fatal fantasy? Fabricated seizures and child abuse. Arch Dis Child April 2002;86:230-233). (Respond: Dr PM Davis, Department of Child Health, Cardiff and Vale NHS Trust, Lansdowne Hospital, Cardiff CF1 8UL, UK).

COMMENT. Seizures are a frequent presenting symptom in Munchausen syndrome by proxy. A closer communication between family physician or pediatrician and epileptologist can help to avoid delay in diagnosis and appropriate management. 